\title{
EFEITOS DO ESTERCO DE CURRAL NA FERTILIDADE DO SOLO, NO ESTADO NUTRICIONAL E NA PRODUÇÃO DA FIGUEIRA ${ }^{1}$
}

\author{
SARITALEONEL $^{2} \&$ ERVAL RAFAEL DAMATTO JUNIOR $^{3}$
}

RESUMO - Foram avaliados os efeitos de doses de esterco de curral na nutrição e produção da figueira (Ficus carica L.), em Botucatu-SP. Os tratamentos corresponderam a doses de esterco de curral: testemunha (sem adubação), 25\%, $50 \%, 75 \%, 100 \%, 125$ $\%$ e $150 \%$ da dose recomendada de N. A avaliação do estado nutricional das plantas de figueira foi realizada através de análises de solo e diagnose foliar no pleno florescimento das plantas. A colheita dos frutos estendeu-se do período de dezembro de 2005 a abril de 2006 e com esses dados determinaram-se a produção e a produtividade. A adubação com esterco de curral foi bastante eficaz para suprir as exigências nutricionais da cultura, uma vez que os teores foliares dos principais macro e micronutrientes se encontravam adequados, indicando resultados positivos com relação à produção de frutos.

Termos para indexação: Ficus carica L.; adubação orgânica; nutrição mineral; produção.

\section{EFFECTS OF COAT MANURE IN THE SOIL FERTILITY, PLANTS NUTRITION AND YIELD OF FIG ORCHARDS}

\begin{abstract}
The effects of coat manure rates in fig trees (Ficus carica L.), nutrition and yield were evaluated in Botucatu-SP. The treatments were constituted by coat manure rates: control, $25 \%, 50 \%, 75 \%, 100 \%, 125 \%$ and $150 \%$ of the recommended nitrogen rate. The nutrition of fig plants was evaluated by soil samples and foliar analyses in the blooming. The fruits harvest started in December of 2005 and it was over in April of 2006, and with this data, it was determined the production and the productivity. The management with coat manure was very efficient to supply the culture nutritional requirement, once the leaves nutrients levels were good to the culture, indicating positive results in relation to the fruit production.
\end{abstract}

Index terms: Ficus carica L.; organic management; mineral nutrition; production.

\section{INTRODUÇÃO}

A figueira (Ficus carica L.) é cultivada desde os tempos mais remotos, porém teve sua importância econômica iniciada no Estado de São Paulo, nos últimos sessenta anos (Amaro, 1972). Conforme dados estatísticos, a área colhida com a cultura da figueira no Brasil, em 2004, foi de 3.130 ha, sendo os Estados do Rio Grande do Sul e São Paulo considerados os maiores produtores. São Paulo é o segundo produtor nacional de figo, com um volume de 7 mil toneladas (Instituto Brasileiro de Frutas, 2005), concentrada na região de Campinas, que responde por todas as exportações brasileiras de figo maduro.

O conhecimento do correto manejo do pomar, apropriado à região produtora, bem como aspectos de adubação e nutrição mineral figuram como informações relevantes de sucesso, não apenas nos novos campos de cultivo, como também nos já existentes. De acordo com Almeida \& Silveira (1997), em observações práticas, foi demonstrado que solos areno-argilosos, bem drenados e com bom teor de matéria orgânica, são os que apresentam melhores características agronômicas para o adequado desenvolvimento da figueira.

A figueira, como todas as espécies vegetais, demanda certa quantidade de nutrientes para fazer crescer seus ramos, folhas, raízes, tronco e para sustentar a produção de frutas. Embora as demandas nutricionais sejam variáveis em função do estado fisiológico da planta e das retiradas de ramos e frutos, a falta de qualquer um dos elementos essenciais pode ser limitante para a cultura. No tocante a este tema, os dados disponíveis na literatura são bastante escassos, no entanto demonstram que a fruteira responde bem a adubações orgânicas, nitrogenadas e fosfatadas. Hiroce et al. (1979) informaram que os nutrientes presentes em maior quantidade nos frutos de figueira são o nitrogênio e o potássio.

Fernandes \& Buzetti (1999) reportam que a figueira cultivada em São Paulo, sob regime de poda drástica anual, apresenta porte arbustivo e, nesse caso, o manejo das características químicas dos solos cultivados com figueira é muito pouco estudado. Os poucos relatos disponíveis versam sobre o uso da adubação orgânica como prática favorável, tanto no desenvolvimento como na produção das figueiras. De acordo com Penteado (1986), fatores de nutrição mineral equilibrados e satisfatórios durante a fase de formação de plantas garantem boas safras na fase produtiva da planta.

Sobre o emprego da matéria orgânica nos cultivos agrícolas, Miysaka \& Okamoto (1992) relatam que a matéria orgânica proporciona mudanças nas características físicoquímicas do solo, como densidade, retenção de água, textura, estrutura, porosidade e condutibilidade térmica, e atua aumentando a capacidade de troca catiônica (CTC), a soma de bases, além de promover uma ação quelante, evitando que alguns nutrientes necessários às plantas se insolubilizem.

${ }^{1}$ (Trabalho 125-07). Recebido em: 15-05-2007. Aceito para publicação: 11-03-2008. Trabalho financiado pela FAPESP (Processo ${ }^{\circ}$ 01/04398-1). ${ }^{2}$ Prof. Dr. do Departamento de Produção Vegetal - Faculdade de Ciências Agronômicas - UNESP, Botucatu-SP. E-mail: sarinel@fca.unesp.br. ${ }^{3}$ Eng. Agr. Doutorando da Faculdade de Ciências Agronômicas - UNESP, Botucatu-SP. E-mail: ervaljr@fca.unesp.br. Bolsista Fapesp. 
Diante do exposto, os objetivos do presente trabalho foram avaliar os efeitos de doses de esterco de curral na nutrição e produção da figueira (Ficus carica L.).

\section{MATERIAL E MÉTODOS}

O trabalho foi conduzido na Faculdade de Ciências Agronômicas/UNESP, em Botucatu-SP (22 $52^{\prime}$ S, 48 $26^{\prime}$ W e altitude $786 \mathrm{~m}$ ), com clima temperado quente (mesotérmico), com chuvas no verão e seca no inverno (Cwa - Koppen) e temperatura média mais quente superior a $22^{\circ} \mathrm{C}(\mathrm{Cunha}$ et al., 1999). O solo da área foi classificado como Nitossolo Vermelho, segundo os critérios da Embrapa (1999). As características químicas iniciais do solo, na camada de 0 a $20 \mathrm{~cm}$, foram as seguintes: $\mathrm{pH}\left(\mathrm{CaCl}_{2}\right)$ $=4,2 ; \mathrm{MO}=24 \mathrm{~g} \mathrm{dm}^{-3} ; \mathrm{P}($ resina $)=3 \mathrm{mg} \mathrm{dm}^{-3} ; \mathrm{H}+\mathrm{Al}=84 \mathrm{mmol}_{\mathrm{c}}$ $\mathrm{dm}^{-3} ; \mathrm{K}=1,4 \mathrm{mmol} \mathrm{dm}^{-3} ; \mathrm{Ca}=12 \mathrm{mmol} \mathrm{dm}^{-3} ; \mathrm{Mg}=5 \mathrm{mmol} \mathrm{dm}^{-}$ 3; $\mathrm{SB}=18 \mathrm{mmol}_{\mathrm{c}} \mathrm{dm}^{-3} ; \mathrm{CTC}=102 \mathrm{mmol}_{\mathrm{c}} \mathrm{dm}^{-3} ; \mathrm{V}=18 \%$, as quais foram analisadas e determinadas de acordo com a metodologia de Raij \& Quaggio (1983).

A área do experimento foi preparada dois meses antes do plantio, realizando aração, seguida de calagem em área total, conforme a análise de solo, objetivando elevar a saturação de bases a $70 \%$ (Raij et al., 1997), utilizando calcário dolomítico. O plantio das mudas de figueira foi realizado em setembro de 2001, com mudas de figueira 'Roxo de Valinhos', adotando espaçamento de $3 \mathrm{~m}$ entre linhas e $2 \mathrm{~m}$ entre plantas, representando uma densidade de $1.660 \mathrm{plantas} / \mathrm{ha}$. O plantio foi feito seguindo as curvas de nível do terreno, e as dimensões das covas foram de $60 \times 60 \times 60 \mathrm{~cm}$, nas quais foram adicionados 20 litros de esterco de curral, $1 \mathrm{~kg}$ de calcário e $1 \mathrm{~kg}$ de termofosfato magnesiano contendo $0,10 \%$ de boro e $0,25 \%$ de zinco. As plantas foram podadas no mês de julho dos anos de 2002 a 2005, com a finalidade de adquirirem a conformação de 12 ramos produtivos, que é considerada a mais recomendada para a cultura.

Os tratamentos, correspondentes aos níveis de adubação orgânica com esterco de curral curtido, foram ministrados nos meses de agosto e setembro dos anos de 2002 a 2005, com metade da dose em cada mês, empregando-se quantidades crescentes: testemunha (sem adubação), $25 \%, 50 \%, 75 \%, 100 \%, 125 \%$ e $150 \%$ da dose recomendada, sendo essas doses calculadas de acordo com o teor de nitrogênio presente no esterco (Tabela 1).

Na Tabela 2, são apresentados os teores de nutrientes presentes nos estercos empregados para as adubações das figueiras. Além da adubação orgânica, realizou-se, em todos os tratamentos, a adubação complementar com termofosfato, aplicando-se $60 \mathrm{~g}$ de $\mathrm{P}_{2} \mathrm{O}_{5}$ no $1^{\circ} \mathrm{e} 2^{\circ}$ anos e $100 \mathrm{~g} \mathrm{de} \mathrm{P}_{2} \mathrm{O}_{5}$ no $3^{\circ} \mathrm{e}$ $4^{\circ}$ anos, além de $60 \mathrm{~g} \mathrm{de} \mathrm{K}_{2} \mathrm{O}$ no $1^{\circ}$ e $2^{\circ}$ anos e $40 \mathrm{~g} \mathrm{de} \mathrm{K}_{2} \mathrm{O}$ no $3^{\circ}$ e $4^{\circ}$ anos.

Utilizou-se o delineamento experimental em blocos casualizados, com 4 repetições, sendo cada parcela experimental representada por 5 plantas úteis. Realizou-se a análise de variância e, quando houve significância, a análise de regressão.

A avaliação do estado nutricional das plantas de figueira foi feita através de diagnose foliar realizada no mês de dezembro/ 2005 (pleno florescimento e início de frutificação). Para a realização da análise foliar, foram colhidas folhas completas recém-maduras, da porção mediana do ramo, nos diferentes lados da planta, com boa exposição ao sol. Cada amostra foi composta de 40 folhas por unidade experimental, coletando-se assim quatro folhas por planta. A determinação dos teores dos nutrientes $\mathrm{N}, \mathrm{P}, \mathrm{K}, \mathrm{Ca}$, $\mathrm{Mg}, \mathrm{S}, \mathrm{B}, \mathrm{Cu}, \mathrm{Fe}, \mathrm{Mn}$ e $\mathrm{Zn}$ foi realizada seguindo a metodologia proposta por Malavolta et al. (1997).

Para as análises de solo, foram feitas amostragens no mês de dezembro/2005, na projeção da copa (faixa adubada), na profundidade de $0-20 \mathrm{~cm}$, coletando-se uma subamostra a cada 2 plantas, num total de 20 por unidade experimental, a fim de constituir uma amostra composta. Os teores de $\mathrm{P}, \mathrm{K}, \mathrm{Ca}, \mathrm{Mg}, \mathrm{pH}$ e $\mathrm{V} \%$ foram determinados segundo a metodologia descrita por Raij et al. (1983).

A colheita anual dos frutos maduros estendeu-se do período de dezembro de 2005 a abril de 2006 , e a produção por planta $(\mathrm{kg})$ foi estabelecida pela multiplicação do número de frutos pelo peso médio dos mesmos. Sabendo-se da lenta decomposição da matéria orgânica no solo, são apresentados somente os dados de produção após quatro anos de cultivo da figueira com adubação com esterco.

\section{RESULTADOS E DISCUSSÃO}

Os resultados de análise de solo para macronutrientes na camada de $0-20 \mathrm{~cm}$ de profundidade, no mês de dezembro/ 2005, encontram-se na Tabela 3, onde é possível observar que os tratamentos não influenciaram significativamente seus teores no solo cultivado com figueira e adubado com doses de esterco de curral. Os valores de $\mathrm{pH}$ e da saturação por bases determinados foram considerados médios no solo da área experimental, pois, de acordo com Raij \& Quaggio (1983), esses teores variam de 5,1 a 5,5 e de 61 a $70 \%$, respectivamente. Os teores de fósforo, potássio, cálcio e magnésio, determinados no solo, foram de 34

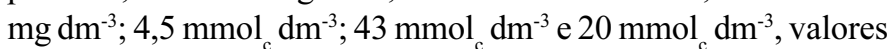
esses considerados altos por Raij \& Quaggio (1983), que relatam valores adequados entre $31 \mathrm{a} 60 \mathrm{mg} \mathrm{dm}^{-3} ; 3,1 \mathrm{a} 6,0 \mathrm{mmol}_{\mathrm{c}} \mathrm{dm}^{-3} ;>$ $7 \mathrm{mmol}_{\mathrm{c}} \mathrm{dm}^{-3} \mathrm{e}>8 \mathrm{mmol}_{\mathrm{c}} \mathrm{dm}^{-3}$, respectivamente. Dessa forma, esses valores são considerados adequados para a cultura da figueira, corroborando os resultados encontrados para o teor de elementos minerais presentes nas folhas (Tabela 5).

Pela Tabela 4, é possível verificar os teores médios de micronutrientes para cada tratamento. Diferentemente do ocorrido com os macronutrientes, os micros apresentaram resposta quadrática aos tratamentos aplicados para cobre e ferro, enquanto para o zinco ocorreu diminuição linear $(y=-0,3929 x+$ 5,$\left.7571 ; \mathrm{r}^{2}=0,69\right)$ em função das doses de esterco aplicadas.

Para o cobre, ajustou-se a seguinte equação: $y=0,0833 \mathrm{x}^{2}-0,7952 \mathrm{x}$ $+7,6857\left(r^{2}=0,61\right)$ e para o ferro: $y=0,4405 x^{2}-2,8452 x+28\left(r^{2}=\right.$ $0,60)$.

Avaliaram-se os teores nutricionais foliares no momento do florescimento, objetivando verificar alguma deficiência que pudesse comprometer a produção, uma vez que há padrões gerais para a cultura. Os teores de nutrientes na massa seca foliar da figueira mostraram a seguinte ordem de concentração no florescimento das plantas: $\mathrm{N}>\mathrm{K}>\mathrm{Ca}>\mathrm{Mg}>\mathrm{P}>\mathrm{S}$, estando seus valores médios apresentados na Tabela 5 . 
Sobre os teores nutricionais em folhas de figueira, existem poucos trabalhos, mas as referências são maiores que nos frutos. Resultados sobre absorção e exportação de nutrientes pela figueira foram apresentados por Hiroce et al. (1979). De acordo com tais resultados, os macronutrientes predominantes nos frutos são: nitrogênio e potássio, enquanto, dentre os micronutirentes, o molibdênio foi o elemento mais encontrado nos frutos.

Através da Tabela 5, observa-se que não houve diferenças para os teores da maior parte dos macronutrientes nas folhas de figueiras adubadas com doses de esterco de curral. Contudo, observou-se resposta quadrática para os teores de nitrogênio (y $\left.=0,2619 x^{2}-2,0238 x+36 ; r^{2}=0,80\right)$ e para os teores de fósforo no solo $\left(y=0,019 x^{2}-0,1952 x+3,2143 ; r^{2}=0,66\right)$.

Os resultados de análise foliar obtidos indicaram que os teores de nitrogênio presente nas folhas se encontravam superiores ao padrão indicado para a cultura (Raij et al., 1997). Já os teores foliares de fósforo, potássio e enxofre estão dentro da faixa considerada adequada, enquanto o cálcio e o magnésio apresentaram teores abaixo desta faixa, porém não se observaram sintomas de deficiência destes nutrientes. Assim, é possível inferir que a adubação orgânica adicionada na forma de esterco de curral curtido foi suficiente para suprir as exigências nutricionais para as plantas.

A adição de nitrogênio diminuiu os teores de cálcio nas folhas, ainda que os teores deste elemento $(\mathrm{Ca})$ no solo estivessem em níveis considerados adequados. Devido aos baixos teores de cálcio nas folhas, é possível sugerir que a aplicação de calcário foi suficiente para neutralizar a acidez do solo; mas, ao longo dos 4 anos do experimento, não foi suficiente para fornecer cálcio para as plantas, que é um nutriente essencial para a boa frutificação da figueira. Tal sugestão é feita baseada nos resultados obtidos para o número médio de frutos por planta e, conseqüentemente, na produção, que é considerada inferior à obtida em cultivos com adubação convencional.

Haag et al. (1979), trabalhando com plantas de figueira conduzidas sob solução nutritiva completa, relataram que as mesmas apresentaram os seguintes teores nutricionais: $\mathrm{N}=33$; $\mathrm{P}=1,7 ; \mathrm{K}=28 ; \mathrm{Ca}=19 ; \mathrm{Mg}=6,6 ; \mathrm{e} \mathrm{S}=2,1 \mathrm{~g} / \mathrm{Kg}$. Os teores associados às deficiências dos referidos elementos foram os seguintes: $\mathrm{N}=24,5 ; \mathrm{P}=0,9 ; \mathrm{K}=1,8 ; \mathrm{Ca}=8 ; \mathrm{Mg}=1,1$ e $\mathrm{S}=1,2 \mathrm{~g} / \mathrm{Kg}$. Dessa maneira, é possível inferir que não houve limitações em elementos nutrientes para o estágio de crescimento da figueira, avaliado no presente trabalho.

Para Brizola (2003), os teores de macronutrientes nas folhas, tanto no decorrer da estação de crescimento das plantas (meses-estações), como entre anos agrícolas consecutivos, representam mais do que apenas diferenças de estágio fisiológico da planta, pois também são influenciadas pelas práticas de adubações, condições de disponibilidade de água e temperaturas. Frente ao exposto, para Mengel e Kirkby (1987), a umidade do solo influencia no suprimento de nutrientes, principalmente para o fósforo e o potássio. Já para Hernandez et al. (1994), diminuições nos teores foliares de P, Mg e K estão mais ligadas a condições de déficit hídrico.

De maneira geral, os tratamentos não influenciaram significativamente nos teores foliares de micronutrientes, como pode ser observado na Tabela 6 . Isso mostra que a concentração de micronutrientes em estercos orgânicos, em especial ao utilizado neste trabalho, é relativamente baixa, não gerando diferenças entre os tratamentos empregados. Os teores de micronutrientes nas folhas seguiram a seguinte ordem de concentração: $\mathrm{Fe}>\mathrm{Mn}>\mathrm{Zn}>\mathrm{B}>\mathrm{Cu}$.

Os teores médios de micronutrientes, no momento do florescimento das plantas, apresentavam-se dentro de uma faixa considerada adequada para a cultura, excetuando-se o ferro e o manganês, que apresentaram teores um pouco abaixo do padrão, sem, contudo, as plantas mostrarem sintomas de sua deficiência.

$\mathrm{O}$ zinco é relatado na literatura como um elemento essencial para promover o crescimento das plantas, sendo este o único micronutriente que apresentou queda significativa em seus teores, nas folhas, em função dos tratamentos $(y=-1,89 x+$ 75,$\left.71 ; \mathrm{r}^{2}=0,49\right)$. Os resultados observados podem sugerir inferências sobre uma diminuição no crescimento das plantas pela falta do elemento, acarretando, consecutivamente, diminuição na produção, uma vez que a figueira, sob regime de poda drástica, produz seus frutos em ramos do ano e normalmente um fruto por nó do ramo. Com menor crescimento e comprimento dos ramos, o número de frutos por planta será diminuído.

A adubação com esterco de curral, de maneira geral, foi considerada eficaz para suprir as exigências nutricionais da cultura, indicando resultados positivos com relação à produção de frutos, onde os tratamentos a influenciaram significativamente, como pode ser observado no Gráfico 1. Neste trabalho, a produtividade média obtida foi de $3,79 \mathrm{~kg}_{\text {planta }}{ }^{-1}$, o que indica uma produtividade média de $6,3 \mathrm{tha}^{-1}$, produtividade essa inferior à média nacional de $8,2 \mathrm{t} \mathrm{ha}^{-1}$, mas que pode servir como base para adoção de sistemas de produção orgânica e integrada na cultura, além da possibilidade de redução nos custos de produção.

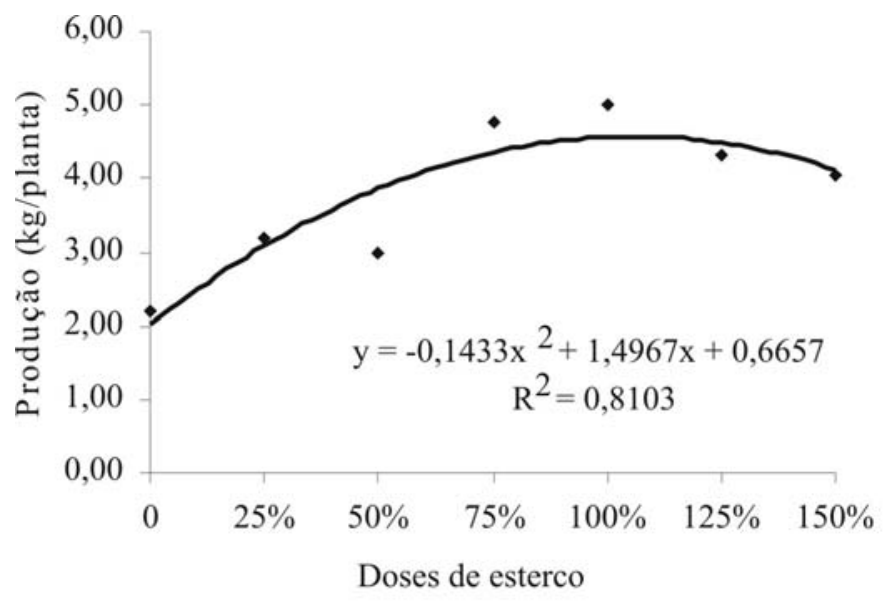

FIGURA 1- Produção $\left(\mathrm{kg} \mathrm{planta}^{-1}\right)$ de plantas de figueira adubadas com doses de esterco de curral (Botucatu-SP abril/2006). 
TABELA 1- Doses de nitrogênio empregados nas adubações anuais (agosto/2002 a agosto/2005). Botucatu, FCA-UNESP, 2006.

\begin{tabular}{ccccccccc}
\hline \multirow{2}{*}{$\begin{array}{c}\text { \% da dose de } \mathrm{N} \\
\text { recomendada }\end{array}$} & \multicolumn{3}{c}{ Níveis de N (g/planta) } & \multicolumn{3}{c}{ Doses de esterco (kg/planta) } \\
\cline { 2 - 8 } & $1^{\circ}$ ano & $2^{\circ}$ ano & $3^{\circ}$ ano & $4^{\circ}$ ano & $1^{\circ}$ ano & $2^{\circ}$ ano & $3^{\circ}$ ano & $4^{\circ}$ ano \\
\hline $0 \%$ & 0 & 0 & 0 & 0 & 0,0 & 0,0 & 0,0 & 0,6 \\
$25 \%$ & 10 & 20 & 30 & 30 & 0,8 & 0,8 & 1,9 & 3,6 \\
$50 \%$ & 20 & 40 & 60 & 60 & 1,6 & 1,6 & 3,8 & 7,3 \\
$75 \%$ & 30 & 60 & 90 & 90 & 2,4 & 2,4 & 5,7 & 11,0 \\
$100 \%$ & 40 & 80 & 120 & 120 & 3,2 & 3,2 & 7,6 & 14,3 \\
$125 \%$ & 50 & 100 & 150 & 150 & 4,0 & 4,0 & 9,5 & 18,0 \\
$150 \%$ & 60 & 120 & 180 & 180 & 4,8 & 4,8 & 11,4 & 22,0 \\
\hline
\end{tabular}

TABELA 2 - Análise química dos estercos de curral curtido utilizados para as adubações das figueiras (1a: agosto/2002; $2^{\mathrm{a}}$ : junho/ 2003; 3a: julho/2004; 4a : julho/2005.). Botucatu, FCA-UNESP, 2006.

\begin{tabular}{cccccccccc}
\hline & \multicolumn{8}{c}{ Resultado em porcentagem na matéria seca } \\
\hline Amostra & $\mathbf{N}$ & $\mathbf{P 2 O}_{\mathbf{2}}$ & $\mathbf{K 2}$ & Umidade & MO & $\mathbf{C}$ & $\mathbf{C a}$ & $\mathbf{M g}$ & $\mathbf{S}$ \\
\hline $1^{\mathrm{a}}$ & 1,26 & 1,52 & 1,68 & 41 & 43 & 23,89 & 1,50 & 0,50 & 0,18 \\
$2^{\mathrm{a}}$ & 2,45 & 2,14 & 1,55 & 37 & 41 & 23,00 & 1,14 & 0,45 & 0,35 \\
$3^{\mathrm{a}}$ & 1,58 & 0,94 & 0,92 & 48 & 39 & 21,70 & 1,29 & 0,84 & 0,21 \\
$4^{\mathrm{a}}$ & 1,12 & 1,20 & 0,80 & 30 & 34 & 18,90 & 0,84 & 0,36 & 0,34 \\
\hline
\end{tabular}

Resultado em mg/kg na matéria seca

\begin{tabular}{cccccccc}
\hline Amostra & $\mathbf{F e}$ & $\mathbf{C u}$ & $\mathbf{M n}$ & $\mathbf{N a}$ & $\mathbf{Z n}$ & $\mathbf{p H}$ & $\mathbf{C} / \mathbf{N}$ \\
\hline $1^{\mathrm{a}}$ & 11300 & 120 & 146 & $\mathrm{~N} / \mathrm{D}$ & 190 & 7,6 & $19 / 1$ \\
$2^{\mathrm{a}}$ & 7950 & 302 & 308 & 1860 & 300 & 7,20 & $9 / 1$ \\
$3^{\mathrm{a}}$ & 14300 & 6 & 5250 & 580 & 286 & 14,00 & $14 / 1$ \\
$4^{\mathrm{a}}$ & 24950 & 156 & 278 & 1460 & 208 & 7,97 & $17 / 1$ \\
\hline
\end{tabular}

Fonte: Laboratório de Análises de Fertilizantes e Corretivos. DRN-FCA.

TABELA 3 - Teores de macronutrientes e valores de pH, M.O., SB, CTC e V\% de solo cultivado com plantas de figueira (Botucatu-SP / dezembro/2005).

\begin{tabular}{|c|c|c|c|c|c|c|c|c|c|}
\hline Tratamentos & $\begin{array}{c}\mathrm{pH} \\
\mathrm{CaCl}_{2} \\
\end{array}$ & $\begin{array}{l}\text { M.O. } \\
\text { g/dm }\end{array}$ & $\begin{array}{c}P \\
\mathbf{m g} / \mathbf{d m}\end{array}$ & $\mathbf{K}$ & Ca & $\begin{array}{c}\mathrm{Mg} \\
\mathbf{m m o l} \mathbf{d} \mathbf{d m}^{3}\end{array}$ & $\begin{array}{l}\text { SB } \\
3 \\
-\end{array}$ & $\begin{array}{c}\text { CTC } \\
---\end{array}$ & $\begin{array}{l}\mathrm{V} \% \\
(\%) \\
\end{array}$ \\
\hline T1: testemunha & 5,7 & 24 & 45 & 4,5 & 51 & 22 & 77 & 105 & 70 \\
\hline $\mathrm{T} 2: 25 \%$ da dose de $\mathrm{N}$ & 5,2 & 27 & 17 & 4,3 & 38 & 16 & 59 & 96 & 53 \\
\hline T3: $50 \%$ da dose de $\mathrm{N}$ & 5,2 & 27 & 24 & 4,4 & 38 & 17 & 60 & 94 & 57 \\
\hline T4: $75 \%$ da dose de $\mathrm{N}$ & 5,5 & 24 & 37 & 4,9 & 41 & 19 & 65 & 92 & 67 \\
\hline T5: $100 \%$ da dose de $\mathrm{N}$ & 5,9 & 23 & 52 & 4,8 & 50 & 24 & 79 & 102 & 74 \\
\hline T6: $125 \%$ da dose de $\mathrm{N}$ & 5,5 & 23 & 37 & 4,4 & 38 & 19 & 61 & 90 & 66 \\
\hline T7: $150 \%$ da dose de $\mathrm{N}$ & 5,8 & 25 & 27 & 4,6 & 44 & 21 & 70 & 94 & 73 \\
\hline Médias & 5,5 & 25 & 34 & 4,5 & 43 & 20 & 67 & 96 & 66 \\
\hline CV $(\%)$ & 7 & 17 & 55 & 21 & 31 & 29 & 29 & 15 & 18 \\
\hline
\end{tabular}

Médias seguidas por letras distintas na coluna diferem pelo teste de Tukey, a 5\% de probabilidade. 
TABELA 4 - Teores médios de micronutrientes no solo cultivado com plantas de figueira, adubadas com doses de esterco de curral (Botucatu-SP-dezembro/2005).

\begin{tabular}{lccccc} 
Tratamentos & Boro & Cobre & $\begin{array}{c}\text { Ferro } \\
\text { mg/dm }\end{array}$ & Manganês & Zinco \\
\hline T1: testemunha & 0,37 & 7,3 & 26 & 7,7 & 5,2 \\
T2: $25 \%$ da dose de N & 0,47 & 5,9 & 23 & 8,6 & 4,9 \\
T3: $50 \%$ da dose de N & 0,54 & 6,1 & 23 & 5,1 & 5,4 \\
T4: $75 \%$ da dose de N & 0,36 & 5,7 & 27 & 8,3 & 3,2 \\
T5: $100 \%$ da dose de N & 0,39 & 6,3 & 22 & 6,5 & 4,2 \\
T6: $125 \%$ da dose de N & 0,37 & 5,8 & 27 & 8,2 & 3,6 \\
T7: $150 \%$ da dose de N & 0,23 & 6,1 & 30 & 9,7 & 2,8 \\
\hline Médias & 0,39 & 6,2 & 25 & 7,7 & 4,2 \\
\hline CV (\%) & 36 & 11 & 15 & 26 & 41 \\
\hline
\end{tabular}

Médias seguidas por letras distintas na coluna diferem pelo teste de Tukey,a $5 \%$ de probabilidade.

TABELA 5 - Teores médios de macronutrientes encontrados em folhas de figueira, adubadas com doses de esterco de curral (Botucatu-SP - dezembro/2005).

\begin{tabular}{lcccccc}
\hline Tratamentos & Nitrogênio & Fósforo & Potássio & Cálcio & Magnésio & Enxofre \\
\hline T1: testemunha & 34 & $3,0 \mathrm{a}$ & 23 & 15 & 4,2 & 2,1 \\
T2: $25 \%$ da dose de N & 33 & $2,9 \mathrm{ab}$ & 22 & 12 & 4,1 & 2,0 \\
T3: $50 \%$ da dose de N & 33 & $2,9 \mathrm{ab}$ & 23 & 12 & 4,2 & 2,2 \\
T4: $75 \%$ da dose de N & 32 & $2,8 \mathrm{ab}$ & 21 & 12 & 4,1 & 2,1 \\
T5: $100 \%$ da dose de N & 32 & $2,5 \mathrm{~b}$ & 23 & 13 & 3,8 & 1,8 \\
T6: $125 \%$ da dose de N & 33 & $2,8 \mathrm{ab}$ & 23 & 13 & 3,8 & 2,2 \\
T7: $150 \%$ da dose de N & 35 & $2,8 \mathrm{ab}$ & 22 & 12 & 4,0 & 2,0 \\
\hline Médias & 33 & 2,8 & 23 & 13 & 4,0 & 2,1 \\
\hline Padrão* & $20-25$ & $1-3$ & $10-30$ & $30-50$ & $7,5-10,0$ & $1,5-3,0$ \\
\hline CV (\%) & 6 & 6 & 5 & 15 & 6 & 11 \\
\hline
\end{tabular}

Médias seguidas por letras distintas na coluna diferem pelo teste de Tukey, a $5 \%$ de probabilidade.

* Fonte: Boletim Técnico 100, IAC, 1997.

TABELA 6 - Teores médios de micronutrientes encontrados em folhas de figueira, adubadas com doses de esterco de curral (Botucatu$\mathrm{SP}-$ dezembro/2005).

\begin{tabular}{lccccc}
\hline Tratamentos & Boro & Cobre & Ferro & Manganês & Zinco \\
\hline T1: testemunha & 37 & 10 & 80 & 64 & 72 \\
T2: $25 \%$ da dose de N & 35 & 10 & 93 & 70 & 77 \\
T3: $50 \%$ da dose de N & 33 & 8 & 80 & 78 & 73 \\
T4: $75 \%$ da dose de N & 31 & 10 & 87 & 66 & 62 \\
T5: $100 \%$ da dose de N & 43 & 11 & 84 & 67 & 62 \\
T6: $125 \%$ da dose de N & 37 & 9 & 82 & 77 & 65 \\
T7: $150 \%$ da dose de N & 32 & 8 & 85 & 95 & 66 \\
\hline Médias & 35 & 9 & 84 & 74 & 68 \\
\hline Padrão* & $30-75$ & $2-10$ & $100-300$ & $100-350$ & $50-90$ \\
\hline CV (\%) & 18 & 23 & 11 & 29 & 13 \\
\hline
\end{tabular}

Médias seguidas pelas mesmas letras na coluna não diferem pelo teste de Tukey, a 5\% de probabilidade.

* Fonte: Boletim Técnico 100, IAC, 1997. 


\section{CONCLUSÃO}

A adubação com esterco de curral foi eficaz para suprir as exigências nutricionais da figueira em formação, uma vez que os teores foliares dos principais macro e micronutrientes se encontravam adequados para a cultura, indicando resultados positivos com relação à produção de frutos, onde a produtividade média foi de $6,3 \mathrm{tha}^{-1}$.

\section{REFERÊNCIAS}

ALMEIDA, M. M. de; SILVEIRA, E. T. da. Tratos culturais na cultura da figueira no Sudoeste de Minas Gerais. Informe Agropecuário, Belo Horizonte, v.18, n.188, p.27-33, 1997.

AMARO, A.A. Uma análise da comercialização de figo em São Paulo. 1972. 71f. Tese (Doutorado em Economia Aplicada) - Escola Superior de Agricultura “Luiz de Queiroz", Universidade de São Paulo, Piracicaba, 1972.

BRIZOLA, R. M. De O. Níveis de adubação orgânica na cultura da figueira. 2003. 78f. Dissertação (Mestrado em Agronomia/ Horticultura). Faculdade de Ciências Agronômicas, Universidade Estadual Paulista Botucatu, Botucatu, 2003.

CUNHA, A.R.; KLOSOWSKI, E.S.; GALVANI, E.; SCOBEDO, J.F.; MARTINS, D. Classificação climática para o município de Botucatu-SP, segundo Koppen. In: SIMPÓSIO EM ENERGIA NA AGRICUlTURA, 1., 1999, Botucatu. Anais... Botucatu: Faculdade de Ciências Agronômicas, Universidade Estadual Paulista, 1999. p.487-491.

EMBRAPA. Centro Nacional de Pesquisa de Solos. Sistema brasileiro de classificação de solos. Rio de Janeiro, 1999. 412 p. FERNANDES, F.M., BUZETTI, S. Fertilidade do solo e nutrição da figueira. In: CORRÊA, L. de S., BOLIANI, A.C. Cultura da figueira: do plantio à comercialização. Ilha Solteira: FUNEP/ FAPESP, 1999. p.69-85.

HAAG, H. P. et al. Distúrbios nutricionais em figueira (Ficus carica L.) cultivada em solução nutritiva. O Solo, Piracicaba, v.71, n.1, p.31-34, 1979.
HERNANDEZ, F.B.T. et al. Resposta da figueira (Ficus carica L.) ao uso da irrigação e nitrogênio na região de Ilha Solteira. Scientia Agrícola, Piracicaba, v.51, n.1, p.99-104, 1994.

HIROCE, R., OLIMA, M., GALLO, JR. Composição mineral e exportação de nutrientes pelas colheitas de frutos subtropicais e temperados. In: CONGRESSO BRASILEIRO DE FRUTICULTURA, 5., 1979, Pelotas. Anais... Pelotas: Sociedade Brasileira de Fruticultura, 1979. p. 179-189.

INSTITUTO BRASILEIRO DE FRUTAS. Anuário brasileiro da fruticultura. Santa Cruz do Sul: Editora Gazeta Santa Cruz, 2005. $136 \mathrm{p}$.

MALAVOLTA, E.; VITTI, G. C.; OLIVEIRA, S. A. Avaliação do estado nutricional das plantas: princípios e aplicações. 2. ed. Piracicaba: Associação Brasileira de Potassa e do Fósforo, 1997. $319 \mathrm{p}$.

MENGEL, K., KIRKBY, E.A. Principles of plant nutrition. $4^{\text {th }}$ ed. Bern: International Potash Institute, 1987. 655p.

MIYSAKA, S., OKAMOTO, H. Importância da matéria orgânica na agricultura. In: ENCONTRO SOBRE MATÉRIAORGÂNICA DO SOLO: PROBLEMAS E SOLUÇÕES; 1992. Botucatu: Faculdade de Ciências Agronômicas/UNESP. P. 01.

PENTEADO, S. R. Fruticultura de clima temperado em São Paulo. Campinas: Fundação Cargil, 1986. p.115-129.

RAIJ, B. van; CANTARELLA, H.; QUAGGIO, J.A.; FURLANI, A.M.C. (Ed). Recomendações de adubação e calagem para o Estado de São Paulo. 2.ed. rev. e atual. Campinas: Instituto Agronômico/Fundação IAC, 1997. 285p. (Boletim Técnico, 100).

RAIJ, B. van,; QUAGGIO, J. A. Métodos de análise de solo para fins de fertilidade. Campinas: Instituto Agronômico de Campinas, 1983. (Boletim Técnico, 81). 